\title{
Enhanced vortex damping by eddy currents in superconductor-semiconductor hybrids
}

\author{
M. Danckwerts ${ }^{1}$, A.R. Goñi ${ }^{1}$, C. Thomsen ${ }^{1}$, K. Eberl ${ }^{2}$, and A.G. Rojo ${ }^{3}$ \\ ${ }^{1}$ Institut für Festkörperphysik, Technische Universität Berlin, Hardenbergstr. 36, 10623 \\ Berlin, Germany \\ ${ }^{2}$ Max-Planck-Institut für Festkörperforschung, Heisenbergstr. 1, 70569 Stuttgart, \\ Germany \\ ${ }^{3}$ Dept. of Physics, University of Michigan, Ann Arbor, MI 48109, USA
}

\begin{abstract}
An enhancement of vortex-motion damping in thin $\mathrm{Pb} / \mathrm{In}$ superconducting films is obtained through coupling to an adjacent twodimensional electron gas formed in a modulation-doped GaAs/AlGaAs heterostructure. This effect is observed by monitoring the power dissipation at the superconductor in the vortex state while increasing the density of the electron gas using a gate voltage. Quantitative agreement is found with calculations based on a viscous model of vortex damping which considers generation of eddy currents in the electron gas by moving flux lines. In the regime of filamentary and channel vortex flow, eddy-current damping leads to striking dissipation breakdown due to stopping of entire vortex channels.
\end{abstract}

71.10.Ca, 73.50.-h, 74.60.Ge, 74.76.Db

Typeset using REVTEX 
Superconductor-semiconductor hybrid structures are emerging as key devices in the search for new physical phenomena resulting from interactions between two systems with dissimilar electronic propertiest. In particular, Josephson-type junctions with $\mathrm{Nb}$ electrodes coupled by a two-dimensional electron gas (2DEG) in InAs layers exhibit phase-sensitive transport due to Andreev reflections of quasi particles at the interfaces between normal metal and superconductor 2 目. Other experiments concentrate on commensurability and interference effects on electron ballistic transport in the 2DEG, which occur when a perpendicular magnetic field is spacially modulated by the vortices of an adjacent superconducting film. In this case, a pronounced suppression of the Hall effect was observed and ascribed to electron diffraction by flux quanta目.

Few investigations are concerned with the influence of a normal metal on the vortex dynamics under a transport current, although the devices were intentionally designed to have no viscous couplinge. One can think of our hybrid system as a modified Giaever's de transformer 7 , in which one of the superconducting films has been replaced by a $2 \mathrm{D}$ electron gas. Under the action of a Lorentz force the vortices move at constant velocity due to viscous damping. For an isolated superconductor, this damping originates from the voltage induced across the normal core of each moving vortex. By bringing a highly mobile electron gas close enough to the superconducting film, i.e. at a distance of the order of the London penetration depth, an additional dissipation mechanism is introduced through magnetic coupling resulting in an increase of viscosity. An interesting issue is to what extent this would affect filamentary and channel vortex flow, for which dissipation jumps are observed in the current-voltage curves 10 . The study of vortex damping in hybrids may provide further insight into vortex-vortex interactions and pinning effects.

This Letter reports the first observation of damping enhancement for vortex motion due to the presence of a high-mobility electron gas in superconductorsemiconductor hybrids. The samples used in our experiments consist of thin $\mathrm{Pb} / \mathrm{In}$ 
films evaporated on top of modulation-doped GaAs/AlGaAs heterostructures. The evidence is found in the decrease of dissipation voltage measured at the superconducting film due to a higher viscosity for vortex flow in the hybrid system, as compared to the case without the 2 DEG beneath. We vary the normal metal conductivity by increasing the carrier density using a gate voltage applied between the 2DEG and a back contact. Our results are in quantitative agreement with the predictions of a model which accounts for the generation of eddy currents in the electron gas by flowing vortices. For an estimated increase in electron density of up to $20 \%$ the relative change observed in dissipation voltage lies in the one-percent range. For the currents and magnetic fields at which filamentary vortex flow occurs, however, striking dissipation reductions are readily achieved.

The semiconductor component of the hybrid system is either a $25 \mathrm{~nm}$ wide GaAs/AlGaAs single quantum well (SQW) or a single heterointerface (SHI) structure. A two-dimensional electron gas is realized by modulation doping and is buried at a distance $D=75 \mathrm{~nm}$ and $50 \mathrm{~nm}$ from the surface for the SQW and SHI structure, respectively 11 . The nominal mobilities and carrier densities of both samples at $4.2 \mathrm{~K}$ and under illumination are $\mu \simeq 8 \times 10^{5} \mathrm{~cm}^{2} / \mathrm{Vs}$ and $n \simeq 5.6 \times 10^{11} \mathrm{~cm}^{-2}$. The electron gas is contacted from the surface by In alloying in order to apply a gate voltage $U_{g}$ between it and a metallic back contact. The variation of the $2 \mathrm{D}$ density was examined previously in photoluminescence experiment\$1 2 . A linear increase in the carrier density $n_{2 \mathrm{D}}$ between its nominal value and at most $\sim 6.5 \times 10^{11} \mathrm{~cm}^{-2}$ can be achieved by applying a gate voltage between 0 and $200 \mathrm{~V}$. Hence the estimated maximum possible increase of density is less than 20\%. Superconducting films of $\mathrm{Pb}$ with nominally 14 at.\% In were evaporated on the semiconductor surface $\left(4 \times 4 \mathrm{~mm}^{2}\right)$ with film thicknesses $d$ ranging from 60 to $300 \mathrm{~nm}$, as determined using atomic-force microscopy. The superconducting transition in zero field occurs at 7.2 K. For transport experiments, Au leads were pressed against the superconductor film. Current-voltage measurements were performed with standard four-terminal configuration using de currents up to $1.5 \mathrm{~A}$. Experiments were carried out at $4.2 \mathrm{~K}$ 
and low perpendicular magnetic fields $B<0.2 \mathrm{~T}$.

To model the coupling between vortex lattice and electron gas in our hybrid samples we consider the effect on the normal metal of the magnetic field $B$ of a moving vortex with speed $v$. The experimental situation is schematically shown in the inset to Fig. 1. Flowing vortices induce an electric field in the 2DEG that generates eddy currents leading to an additional dissipation which, in turn, forces the fluxoids to slow down. In the limit $D \ll \lambda^{2} / d$ of small superconductor-2DEG distances as compared to the effective London penetration depth, the magnetic field of a vortex can be approximated as $\mathbf{B} \sim\left(\Phi_{0} / 2 \pi \lambda^{2}\right) K_{0}(r / \lambda) \hat{z}$, where $\Phi_{0}=h / 2 e$ is the flux quantum and $K_{0}$ the zeroth-order Bessel function of imaginary argument 13 . For our samples the effective penetration depth is five to eight times $D$ 目. The vector potential in the plane of the 2DEG can be written as

$$
\mathbf{A}(\rho)=\frac{\Phi_{0}}{2 \pi \rho} F(\rho / \lambda) \hat{e}_{\varphi}
$$

where $\rho$ is the polar radius from the vortex core, and $F(x)=\int_{0}^{x} d y y K_{0}(y)$.

The time-varying vector potential produced by a flowing vortex induces an electric field $\mathbf{E}=v \frac{\partial}{\partial x} \mathbf{A}(x-v t, y)$, which causes joule dissipation in the $2 \mathrm{D}$ gas. The energy loss per unit time is calculated according to

$$
\frac{d \varepsilon}{d t}=\sigma_{2 \mathrm{DEG}} v^{2} \int d^{2} x\left[\frac{\partial}{\partial x} \mathbf{A}(\rho / \lambda)\right]^{2} \simeq \frac{\Phi_{0}^{2}}{2 \pi \lambda^{2}} \sigma_{2 \mathrm{DEG}} v^{2} \equiv \eta_{2 \mathrm{DEG}} v^{2}
$$

where $\sigma_{2 \mathrm{DEG}}$ is the conductivity of the $2 \mathrm{D}$ electron gas and the dimensionless integrals are assumed to be of the order of one.

We arrive at the general result that eddy current generation in the hybrid system manifests itself in a contribution to the viscosity $\eta_{2 \mathrm{DEG}}$ describing the enhanced damping of vortex motion. This effect can be observed if $\eta_{2 \mathrm{DEG}}$ is comparable to the viscosity of type-II superconducting material, $\eta_{\mathrm{SC}}=\sigma_{n} d \Phi_{0}^{2} / 2 \pi a^{2} 14$, where $\sigma_{n}$ is the normal state conductivity of the superconductor and $a$ the vortex core radius. The electron gas acts as a shunt conductor, thus increasing the system viscosity $\eta_{\mathrm{tot}}=\eta_{\mathrm{SC}}+\eta_{2 \mathrm{DEG}}$ by a factor 


$$
1+\frac{\eta_{2 \mathrm{DEG}}}{\eta_{\mathrm{SC}}}=1+\left(\frac{a}{\lambda}\right)^{2} \frac{\sigma_{2 \mathrm{DEG}}}{\sigma_{n} d}
$$

Hence the dissipation voltage under a transport current is $U_{d} \propto v \sqrt{14}$ and the vortex velocity is determined by the balance between the driving force $j B$ and the viscous drag $\eta v$. Eddy-current damping grows in proportion to $\sigma_{2 \mathrm{DEG}}$, i.e. to the carrier density $n_{2 \mathrm{D}}$ of the electron gas.

The dissipation due to flowing vortices in the PbIn superconducting film is reduced by increasing the charge density in the neighboring electron gas. Figure 1 shows a typical dissipation voltage $\left(U_{d}\right)$ versus gate bias $\left(U_{g}\right)$ curve of a $\mathrm{PbIn} / \mathrm{SHI}$ hybrid sample measured at a constant transport current of $600 \mathrm{~mA}$. Sweeping $U_{g}$ from 0 to $170 \mathrm{~V}$ causes a linear decrease in dissipation of $\Delta U_{d}=0.022 \mathrm{mV}$, i.e. $\sim 0.1 \%$ of the initial value. Since optical and transport experiments could not be carried out simultaneously, we give here the gate voltage as a measure of the electron density which we assume to vary linearly with $U_{g}$, as inferred from optical measurements12. We emphasize that in spite of the relatively small change in 2DEG density the coupling within the hybrid appears to be effective enough to show up in its dissipative behavior. We also notice that during the experiment no leakage current between superconductor and electron gas was ever detected, thus we rule out any spurious bias to be at the origin of the observed effect.

We interpret the dissipation change in the superconductor with rising charge density in the 2DEG as the effect of eddy currents in the electron gas which slow down the vortices causing the voltage across the superconductor to decrease. Using Eq. (3) we can now estimate the magnitude of this effect. Taking $a \approx \lambda, d \approx$ $100 \mathrm{~nm}, \sigma_{n} \approx 1.4 \times 10^{5} \Omega^{-1} \mathrm{~cm}^{-1}$ as obtained from resistance measurements, and $\sigma_{2 \mathrm{DEG}} \approx 0.08 \Omega^{-1}$ with the $2 \mathrm{DEG}$ parameters given above, this yields $\eta_{2 \mathrm{DEG}} / \eta_{\mathrm{SC}} \approx$ $5 \%$. This change in dissipation voltage corresponds to the difference between having and not having the electron gas next to the superconductor. In our experiments, however, we start from a finite density and produce a variation of about 10\%. Thus, the calculated dissipation change is around $0.5 \%$, in very good agreement with the 
experimental results.

Eddy-current damping effects are much more pronounced in the regime of filamentary and channel vortex flow, for they can lead to a striking fall of dissipation voltage by more than one order of magnitude, as shown in Fig. 2. Here, $U_{d}$ was measured at a current close to the repinning transition of a large vortex channel. The inset to Fig. 2 displays the $I V$ characteristic of the superconducting film measured for a field of $53 \mathrm{mT}$ and at $4.2 \mathrm{~K}$ but without gate bias. The abrupt jumps and large hysteresis apparent in the $I V$ curve are the signature of channel vortex flow 6 . As indicated by the arrow, the point where the measurement of dissipation versus gate voltage was carried out is close above the critical current at which the downward jump occurs. In this case, the vortex speed is just high enough for the channel to keep flowing. Energy loss due to eddy currents slows the vortices further down, so that the whole channel will eventually be repinned. When the vortex channel stops, dissipation suddenly drops. This process is irreversible since, as can be seen in Fig. 2, dissipation does not resume to its initial value when the gate voltage is swept back to zero. This effect is highly reproducible even after heating the sample over $T_{c}$ and re-cooling.

The study of the dependence on gate voltage of the current values at which dissipation jumps occur provides further information about the nature of filamentary vortex flow and the role played by pinning. Figure 3 shows one example in which the currents for the upward and downward voltage jumps are plotted as a function of gate bias (the corresponding $I V$ curve is displayed in the inset to Fig. 3). The jump-up current is independent of the $2 \mathrm{D}$ density indicating that the pinning strength is not appreciably affected by the presence of the electron gas. In contrast, the jump-down current increases with $U_{g}$. With increasing viscosity the vortices of a moving channel slow down such that its repinning occurs at larger values of the transport current. This is interpreted as additional evidence of a repinning force which depends on vortex velocity. A similar increase in jump-down current is observed for thin PbIn 
films on glass by decreasing the external magnetic field 8 .

The influence of magnetic field homogeneity on eddy-current damping is revealed by the percentage change in dissipation as a function of magnetic field at constant transport current. As a measure of the damping strength, the maximum dissipation change $\Delta U_{d}$ for a gate voltage interval of $\Delta U_{g}=170 \mathrm{~V}$ is normalized by the dissipation voltage $U_{d 0}$ at zero bias. The corresponding values measured at $500 \mathrm{~mA}$ and $1000 \mathrm{~mA}$ are shown in Fig. 4 as a function of the external magnetic field $B$. At $B \leq 30 \mathrm{mT}$ damping causes $0.1-0.2 \%$ change in dissipation. At $40 \mathrm{mT}$, the data for both $0.5 \mathrm{~A}$ and $1 \mathrm{~A}$ display a sharp maximum and become very small at fields larger than $50 \mathrm{mT}$, where the resistance of the film is close to normal but the transport behavior is characterized by massive vortex flow.

The weakening of the effect of eddy-current damping on dissipation with increasing magnetic field (solid line in Fig. 4) can be explained as due to the growing homogeneity of the field pattern of the vortices while approaching the upper critical field $B_{c 2}$. At low magnetic fields, i.e. low vortex density, the field distribution is very inhomogeneous, since $B$ has a maximum at the vortex cores dropping to zero between them. Thus, $\dot{B}$ is large and damping is efficient. When vortices start to overlap, the lateral modulation of magnetic field in the plane of the 2DEG is continuously reduced. As a consequence, eddy currents as well as damping effects are weak. In contrast, the peak at $40 \mathrm{mT}$ is associated with the enhancement of the effects due to eddy-current damping in the regime dominated by filamentary vortex flow. Here the dissipation change is greatly enhanced by fluctuations in the number of moving vortices contributing to dissipation due to the depinning and repinning of a large number of small vortex filaments in quick succession.

In summary, we have observed significant additional damping of vortex motion in superconductor-semiconductor hybrid systems. A theoretical model is used to calculate the damping effect from eddy currents generated in the 2D electron gas showing quantitative agreement with the experiment. Under conditions of filamentary vortex 
flow, the energy loss due to eddy currents leads to the stopping of entire channels, such that power dissipation in a hybrid device can be switched off by slightly increasing the electron density. At large fields damping is weak due to the vanishing lateral field modulation in the plane of the 2DEG. We point out that, although our observations can be explained within the framework of classical electrodynamics, novel effects due to quantization of the electron gas conductivity are anticipated to occur for the conditions of the experiments. Our results provide further insight into the issue of vortex dynamics with dissipation and open up a new class of devices for the study of correlations between adjacent non-tunneling systems with dissimilar electronic and magnetic properties.

A.G.R. acknowledges useful conversations with John Clem and Cagliyan Kurdak and partial support from the National Science Foundation. 


\section{REFERENCES}

${ }^{1}$ For recent contributions and references, see Proc. 23th Int. Conf. on the Physics of Semiconductors, Berlin, Germany, July 1996, ed. by M. Scheffler and R. Zimmermann (World Scientific, Singapore, 1996); Proc. 11th Int. Conf. on Electronic Properties of Two-Dimensional Systems, Nottingham, UK, August 1995, Surf. Sci. 361-362, (1996).

${ }^{2}$ C. Nguyen, H. Kroemer, and E. L. Hu, Phys. Rev. Lett. 69, 2847 (1992).

${ }^{3}$ A. Dimoulas, J. P. Heida, B. J. v. Wees, T. M. Klapwijk, W. v. d. Graaf, and G. Borghs, Phys. Rev. Lett. 74, 602 (1995).

${ }^{4}$ B. J. v. Wees, Surf. Sci. 361-362, 293 (1996).

${ }^{5}$ A. K. Geim, S. J. Bending, and I. V. Grigorieva, Phys. Rev. Lett. 69, 2252 (1992);

A. K. Geim, S. J. Bending, I. V. Grigorieva, and M. G. Blamire, Phys. Rev. B 49, R5749 (1994).

${ }^{6}$ G. H. Kruithof, P. C. van Son, and T. M. Klapwijk, Phys. Rev. Lett. 67, 2725 $(1991)$

${ }^{7}$ I. Giaever, Phys. Rev. Lett. 15, 825 (1965).

${ }^{8}$ M. Danckwerts, A. R. Goñi, and C. Thomsen, Phys. Rev. B 59, R6624 (1999).

${ }^{9}$ R. Wördenweber, P. Kes, and C. C. Tsuei, Phys. Rev. B 33, 3172 (1986); S. Bhattacharya and M. J. Higgins, Phys. Rev. B 52, 64 (1995); M. C. Hellerquist, D. Ephron, W. R. White, M. R. Beasley, and A. Kapitulnik, Phys. Rev. Lett. 76, 4022 (1996); W. Henderson and E. Y. Andrei, M. J. Higgins, and S. Bhattacharya, Phys. Rev. Lett. 77, 2077 (1996).

${ }^{10}$ N. Grønbech-Jensen, A. R. Bishop, and D. Domínguez, Phys. Rev. Lett. 76, 2985 (1996).

${ }^{11}$ S. Ernst, A. R. Goñi, K. Syassen,and K. Eberl, Phys. Rev. Lett. 72, 4029 (1994) 
${ }^{12}$ A. R. Goñi, M. Danckwerts, U. Haboeck, K. Eberl and C. Thomsen, phys. stat. sol. (b) 215 (1999)

${ }^{13}$ J. R. Clem, in Inhomogeneous Superconductors, ed. by D. U. Gubser, T. L. Francavilla, S. A. Wolf, and J. R. Leibowitz (American Institute of Physics, New York, 1980), p. 245.

${ }^{14}$ M. Tinkham, Introduction to Superconductivity (McGraw-Hill, 1996), p. 169. 


\section{FIGURES}

Fig. 1. Dissipation voltage as a function of gate bias of a PbIn/SHI hybrid structure in a magnetic field of $53 \mathrm{mT}$ and at $4.2 \mathrm{~K}$. The transport current was 600 $\mathrm{mA}$. The inset shows a sketch of the hybrid sample. $I$ is the transport current. The arrow represents a flux line with magnetic field $B$ moving at a speed $v$.

Fig. 2. Dissipation voltage as a function of gate bias of a $\mathrm{PbIn} / \mathrm{SHI}$ hybrid structure in a perpendicular magnetic field of $53 \mathrm{mT}$ for $500 \mathrm{~mA}$ current and at 4.2 K. The inset shows the corresponding current-voltage characteristic. The point where the measurement of dissipation versus gate voltage was taken is marked with an arrow.

Fig. 3. Dependence on gate voltage of the critical current for the upward (up triangles) and the downward voltage jump (down triangles) for a PbIn/SQW hybrid at $4.2 \mathrm{~K}$ and at a magnetic field of $71 \mathrm{mT}$ within the region of channel vortex flow. The inset shows the corresponding $I V$ characteristic.

Fig. 4. Dissipation percentage change versus magnetic field for a $\mathrm{PbIn} / \mathrm{SQW}$ hybrid sample at $4.2 \mathrm{~K}$. Data were taken at $500 \mathrm{~mA}$ (solid symbols) and $1000 \mathrm{~mA}$ (open symbols). The solid line is a guide to the eye. The shaded area indicates the field range within which voltage jumps are observed for the used currents. 


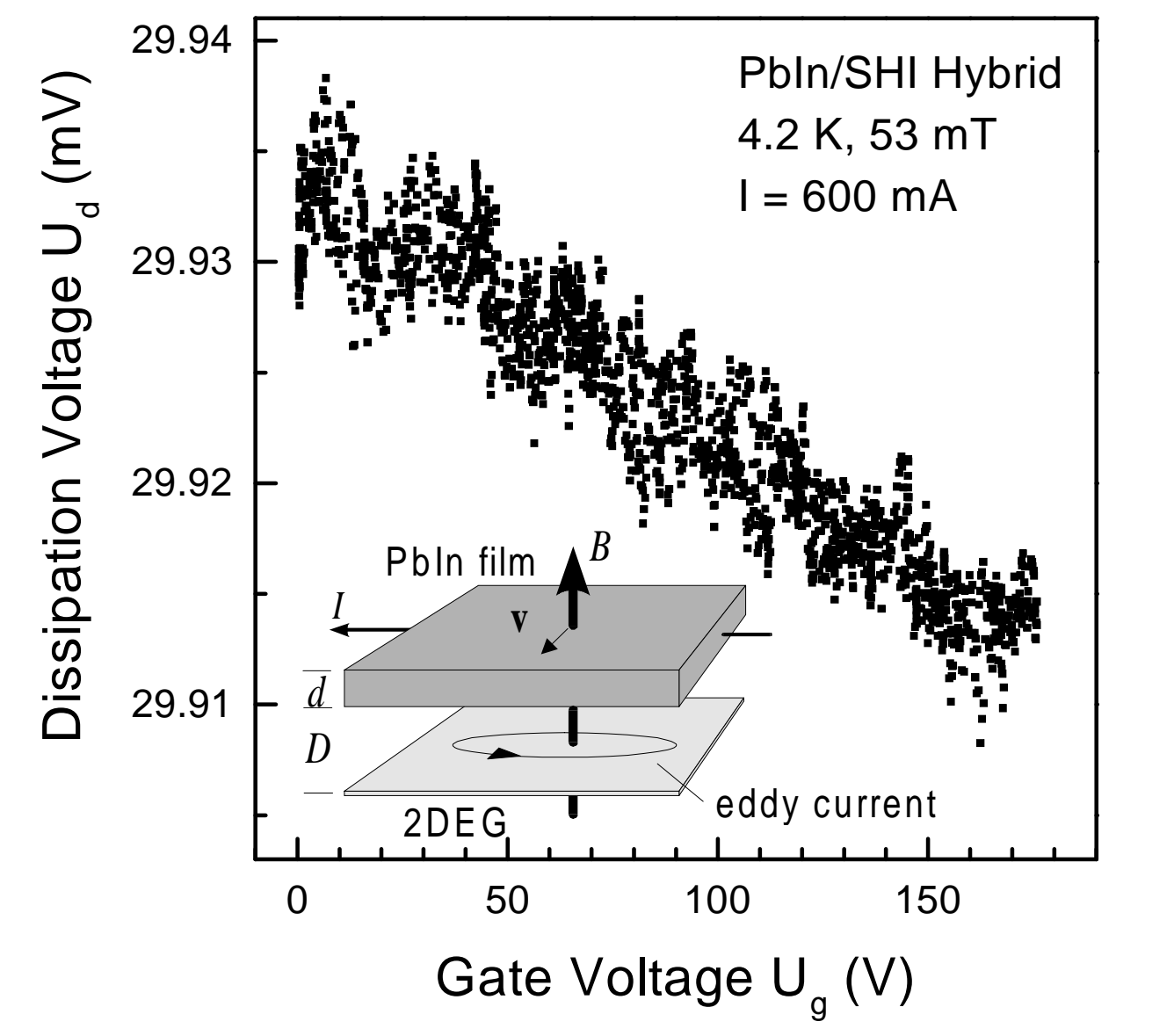

Fig. 1 Danckwerts et al. 


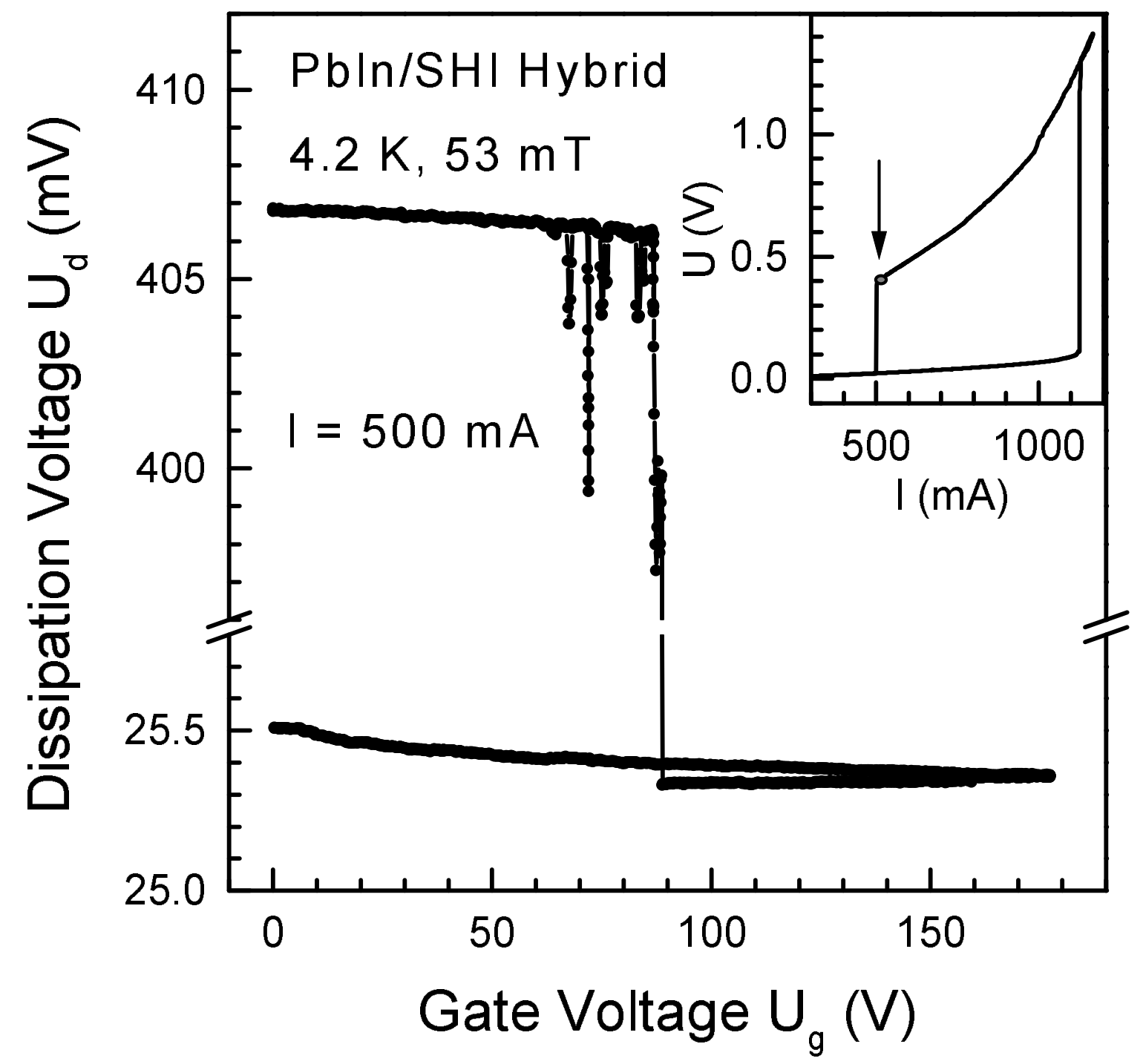

Fig. 2

Danckwerts et al. 


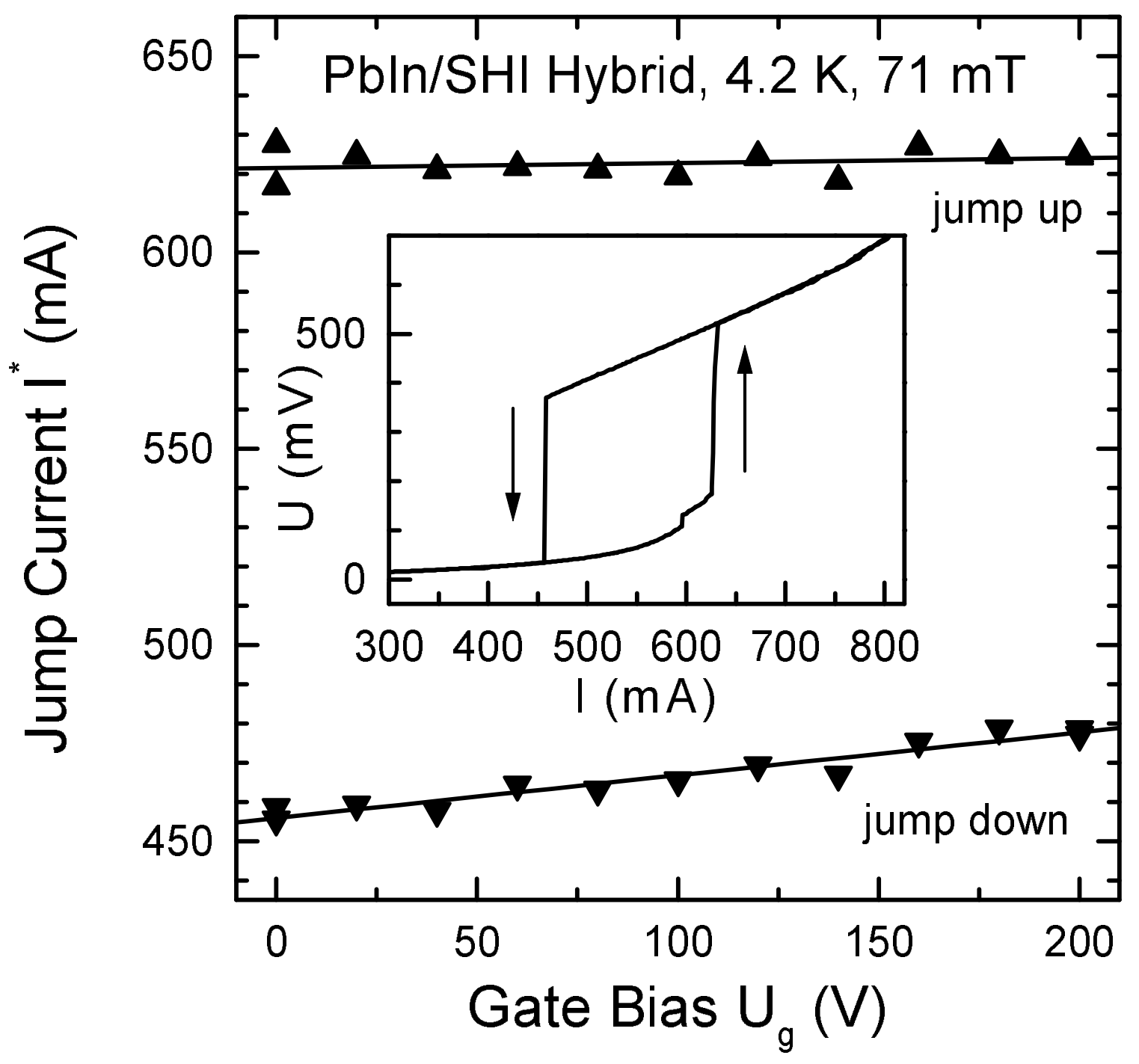

Fig. 3

Danckwerts et al. 


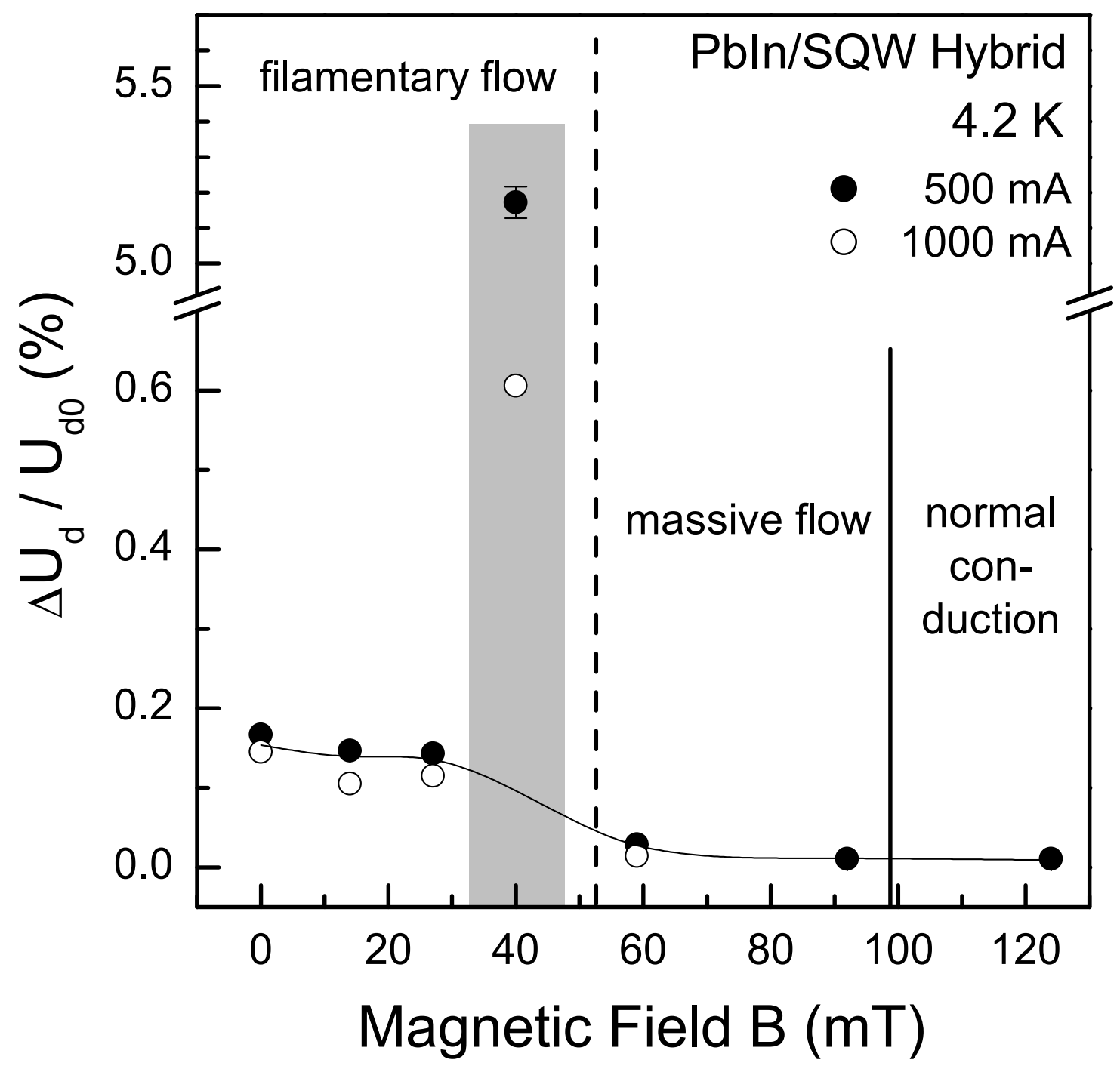

Fig. 4 Danckwerts et al. 